\title{
Mass spectrometry imaging identifies metabolic patterns associated with malignant potential in pheochromocytoma and paraganglioma
}

\author{
Masanori Murakami ${ }^{1, *}$, Na Sun ${ }^{2, *}$, Christian Greunke ${ }^{(1)}{ }^{2}$, Annette Feuchtinger ${ }^{2}$, Stefan Kircher ${ }^{3}$, \\ Timo Deutschbein ${ }^{4,5}$, Thomas Papathomas', Nicole Bechmann ${ }^{8,9,10,11}$, Paal William Wallace ${ }^{8}$, Mirko Peitzsch ${ }^{8}$, \\ Esther Korpershoek ${ }^{12}$, Juliane Friemel ${ }^{13}$, Anne-Paule Gimenez-Roqueplo ${ }^{14,15}$, Mercedes Robledo ${ }^{16}$,

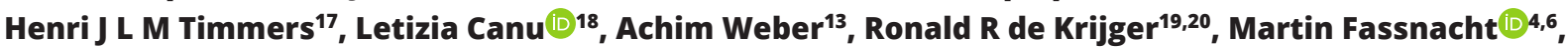

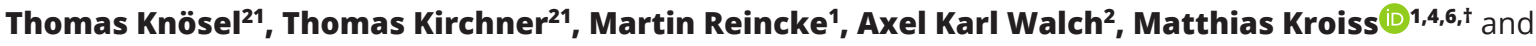 \\ Felix Beuschlein $\mathbb{1} 1,22$, t
}

${ }^{1}$ Medizinische Klinik und Poliklinik IV, Klinikum der Ludwig-Maximilians-Universität München, Munich, Germany, ${ }^{2}$ Research Unit Analytical Pathology, German Research Center for Environmental Health $(\mathrm{GmbH})$, Helmholtz Zentrum München, Neuherberg, Germany, ${ }^{3}$ nstitute for Pathology, University of Würzburg, Würzburg, Germany, ${ }^{4}$ Department of Internal Medicine I, Division of Endocrinology and Diabetology, University Hospital Würzburg, University of Würzburg, Würzburg, Germany, ${ }^{5}$ Medicover Oldenburg MVZ, Oldenburg, Germany, ${ }^{6}$ Comprehensive Cancer Center Mainfranken, University of Würzburg, Würzburg, Germany, Institute of Metabolism and Systems Research, University of Birmingham, Birmingham, UK, ${ }^{8}$ Institute of Clinical Chemistry and Laboratory Medicine, University Hospital Carl Gustav Carus, Medical Faculty Carl Gustav Carus, Technische Universität Dresden, Dresden, Germany, ${ }^{9}$ Department of Medicine III, University Hospital Carl Gustav Carus, Technische Universität Dresden, Dresden, Germany, ${ }^{10}$ Department of Experimental Diabetology, German Institute of Human Nutrition Potsdam-Rehbruecke, Nuthetal, Germany, ${ }^{11}$ German Center for Diabetes Research (DZD), München-Neuherberg, Germany, ${ }^{12}$ Department of Pathology, Erasmus MC Cancer Institute, University Medical Center Rotterdam, Rotterdam, The Netherlands, ${ }^{13}$ Institute for Pathology and Molecular Pathology, Universitätsspital Zürich, Zurich, Switzerland, ${ }^{14}$ Université de Paris, PARCC, INSERM, Equipe labellisée par la Ligue contre le Cancer Paris, France, ${ }^{15} \mathrm{Genetics} \mathrm{department,} \mathrm{AP-HP,} \mathrm{Hôpital} \mathrm{européen} \mathrm{Georges} \mathrm{Pompidou,} \mathrm{Paris,} \mathrm{France,}{ }^{16}$ Hereditary Endocrine Cancer Group, CNIO, Madrid, Spain and Centro de Investigación Biomédica en Red de Enfermedades Raras (CIBERER), Madrid, Spain, ${ }^{17}$ Department of Medicine, Division of Endocrinology, Radboud University Medical Center, Nijmegen, the Netherlands, ${ }^{18}$ Department of Experimental and Clinical Biomedical Sciences 'Mario Serio', University of Florence, Florence, Italy, ${ }^{19}$ Department of Pathology, University Medical Center Utrecht, Utrecht, the Netherlands, ${ }^{20}$ Princess Máxima Center for Pediatric Oncology, Utrecht, the Netherlands, ${ }^{21}$ Institute of Pathology, Ludwig-Maximilians-Universität München, Munich, Germany, and ${ }^{22}$ Department of Endocrinology, Diabetology and Clinical Nutrition, Universitätsspital Zürich, Zurich, Switzerland

*M Murakami and N Sun contributed equally as first authors to Matthias Kroiss

${ }^{\dagger} \mathrm{M}$ Kroiss and $\mathrm{F}$ Beuschlein contributed equally as senior authors

E-mail

Kroiss_M@ukw.de

\begin{abstract}
Objective: Within the past decade, important genetic drivers of pheochromocytoma and paraganglioma (PPGLs) development have been identified. The pathophysiological mechanism that translates these alterations into functional autonomy and potentially malignant behavior has not been elucidated in detail. Here we used MALDImass spectrometry imaging (MALDI-MSI) of formalin-fixed paraffin-embedded tissue specimens to comprehensively characterize the metabolic profiles of PPGLs.

Design and methods: MALDI-MSI was conducted in 344 PPGLs and results correlated with genetic and phenotypic information. We experimentally silenced genetic drivers by siRNA in PC12 cells to confirm their metabolic impact in vitro.

Results: Tissue abundance of kynurenine pathway metabolites such as xanthurenic acid was significantly lower $(P=2.35 \mathrm{E}-09)$ in the pseudohypoxia pathway cluster 1 compared to PPGLs of the kinase-driven PPGLs cluster 2. Lower abundance of xanthurenic acid was associated with shorter metastasis-free survival (log-rank tests $P=7.96 \mathrm{E}-06$ ) and identified as a risk factor for metastasis independent of the genetic status (hazard ratio, 32.6, $P=0.002$ ). Knockdown of
\end{abstract}

(c) 2021 European Society of Endocrinology Printed in Great Britain
Published by Bioscientifica Ltd. 
$S d h b$ and $V h /$ in an in vitro model demonstrated that inositol metabolism and sialic acids were similarly modulated as in tumors of the respective cluster.

Conclusions: The present study has identified distinct tissue metabolomic profiles of PPGLs in relation to tumor genotypes. In addition, we revealed significantly altered metabolites in the kynurenine pathway in metastatic PPGLs, which can aid in the prediction of its malignant potential. However, further validation studies will be required to confirm our findings.

\section{Introduction}

Pheochromocytomas and paragangliomas (PPGLs) are neuroendocrine tumors that arise from the adrenal medulla and from sympathetic paraganglia, respectively. Although the majority of PPGLs follow a benign disease course, up to $20 \%$ of tumors can develop metastatic spread, which defines malignant behavior (1). Over the years, targeted and untargeted genomic studies have revealed that PPGL tumorigenesis is driven mainly by germline or somatic mutations in susceptibility genes comprising SDHA, $S D H B, S D H C, S D H D, S D H A F 2$ (collectively referred to as SDHx), FH, MDH2, SLC25A11 (2), EPAS1, VHL, NF1, HRAS, FGFR1, RET, TMEM127, and MAX $(3,4,5)$. Based on the transcriptomic classification, PPGL tumors can be grouped into three clusters (6). Cluster 1 includes tumors driven by germline or somatic mutations in SDHx, VHL, EPAS1 or genes encoding members of the Krebs cycle pathway $\mathrm{FH}$ and $\mathrm{MDH} 2(6,7)$. In contrast, the cluster 2 subgroup comprises familial or sporadic tumors with underlying RET, NF1, TMEM127, HRAS, KIF1B, and MAX mutations (7). The third cluster is called the Wnt-altered subtype, driven by MAML3 fusions and CSDE1 somatic mutations (5). There is ample evidence that $S D H B / F H$ mutations are associated with a high risk toward metastatic progression $(8,9,10)$. Clinical parameters, including primary tumor size and extra-adrenal location, are further clinical risk factors for malignancy (11).

Analysis of plasma and urinary metanephrine, normetanephrine and 3-methoxytyramine from PPGL patients has demonstrated secretory patterns, which are strongly associated with the underlying genotypes $(12,13)$. A recent quantitative metabolomics study of plasma samples has further identified distinct alterations in metabolic patterns that resolved upon PPGL removal (14). Tissue metabolome profiling of fresh frozen PPGLs has previously been performed with a particular focus on SDH deficiency and provided evidence of higher succinate: fumarate ratios of $S D H x$ mutant vs wild type tumors indicative of impaired Krebs cycle and respiratory chain $(15,16)$. NMR spectroscopy in a small number of frozen tissue homogenates showed that PPGL clusters not only differ in their adenine nucleoside phosphate content but also in guanosine content (17). A more comprehensive picture of tissue metabolic processes, however, is lacking.

Recent advances in MALDI-mass spectrometry imaging (MSI) permit the superimposing of metabolic data with the spatial information conveyed by conventional histology. This technique has recently allowed us to refine the functional anatomy of the human adrenal gland $(18,19)$, has provided novel biomarkers and pathways associated with malignant behavior in adrenocortical carcinoma (20) and has yielded insight into genotype/phenotype correlations of aldosterone-producing adenoma (21). Here, we report the first comprehensive MALDI-MSI study of a large set of PPGLs with rich genetic and clinical information in order to gain further insights into PPGL pathogenesis and clinical outcome of patients with this condition.

\section{Subjects and methods}

\section{Patient cohort}

A total of 344 PPGL tumors were retrieved from 8 specialized European centers: (i) Klinikum der Universität München, Munich, Germany (74 samples), (ii) UniversitätsSpital Zürich, Zurich, Switzerland (69 samples), (iii) University of Würzburg, Würzburg, Germany (60 samples), (iv) Hôpital Européen Georges Pompidou, Paris, France (54 samples), (v) Technische Universität Dresden, Dresden, Germany (29 samples), (vi) University of Florence, Florence, Italy (28 samples), (vii) CNIO, Spain and Centro de Investigación Biomédica en Red de Enfermedades Raras (CIBERER), Madrid, Spain (23 samples), and (viii) University Medical Center Rotterdam, Rotterdam, The Netherlands 
(7 samples). Diagnostic workup was done following established pathways based on clinical, biochemical, and morphological data according to the Endocrine Society Clinical Practice Guidelines (1). Twenty-one samples (21 out of $344 ; 6.1 \%$ ) from 19 patients were considered malignant: In accordance with the Endocrine Society Clinical Practice Guidelines (1), metastatic disease was defined as the presence of metastases in non-chromaffin tissue. The detection of metastasis was based on the combination of imaging studies including functional imaging in 15 patients ( 6 patients with MIBG, 6 patients with FDG-PET, 3 patients with F-DOPA-PET, 2 patients with Ga-DOTATATE PET/CT and 1 with somatostatin receptor scintigraphy) and histopathology after surgical removal in 8 patients. As a total, 17 primary tumors and 4 metastases to other organs were found. All patients had provided written informed consent and the study was approved by ethical committees at all participating institutions. Genetic examination and measurement of urine samples are described in the supplementary methods (see section on supplementary materials given at the end of this article).

\section{Tissue microarrays, immunohistochemistry, and digital image analysis}

Construction of tissue microarrays (TMAs) using formalin-fixed, paraffin-embedded (FFPE) tissues, immunohistochemistry of SDHA and SDHB, and digital image analysis are described in supplementary methods.

\section{MALDI-MSI}

Following tissue sample preparation (see supplementary methods), the MALDI-MSI measurement was performed on a Bruker Solarix 7T FT-ICR-MS (Bruker Daltonics, Bremen, Germany). The MALDI-MSI data were acquired over a mass range of $m / z$ 50-1000. Mass imaging data were acquired in negative ionization mode with $70 \mu \mathrm{m}$ spatial resolution using 50 laser shots at a frequency of $500 \mathrm{~Hz}$. After the MALDI-MSI measurement, the acquired data from the tissue samples underwent spectra processing in FlexImaging v. 4.2 (Bruker Daltonics). Following the MALDI imaging experiments, the matrix was removed with 70\% ethanol. The tissue sections were stained with H\&E and scanned with a digital Mirax Desk slide scanner.

Metabolite annotation was performed using METLIN, http://metlin.scripps.edu/ (22), the Human Metabolome Database (HMDB, http://www.hmdb.ca/) (23) and METASPACE, which is a framework for false discovery rate (FDR)-controlled metabolite annotation at the level of the molecular sum formula for high-mass-resolution imaging mass spectrometry (http://annotate.metaspace2020. $\mathrm{eu} /$ ) (24). Pathway enrichment analysis was applied to identify discriminative features of the mutation status of PPGLs, using Metabolite Set Enrichment Analysis with MetaboAnalyst 3.0 (http://www.metaboanalyst.ca) and the KEGG database (http://www.genome.jp/kegg/). Bioinformatics and statistical analysis are described in supplementary methods. Example images of distinct metabolites across a larger part of the TMA are provided in Supplementary Fig. 1. For assessment of potential confounding factors, 'sample age' was defined by the interval between TMA construction and time of surgery.

Cell culture and siRNA transfection, quantitative realtime PCR, immunoblotting and metabolome analysis of in vitro models are described in supplementary methods.

\section{Results}

\section{Demographics}

The clinical characteristics of the 331 included PPGL patients (153 men/178 women) with a median age at surgery of 50 (40-62) years) who had 344 tumors are summarized and stratified by the evidence of malignant behavior in Table 1. For 188 patients, germline and somatic mutation status were available. Nineteen patients (with 21 tumor samples in total) were diagnosed with malignant PPGLs. Among those, larger tumor size, abdominal localization, low urinary metanephrine concentration and SDHB mutation were associated with the development of metastases (logrank tests $P=2.25 \mathrm{E}-04,6.92 \mathrm{E}-05,5.55 \mathrm{E}-06$ and 6.97E-07, respectively, Supplementary Fig. 2A). Multivariable Cox proportional hazard modeling revealed that larger tumor size and $S D H B$ mutation remained associated with malignant behavior (hazard ratio, 14.0 and $18.1 ; P=0.032$ and 0.018, respectively), while stepwise regression rejected abdominal paraganglioma and lower urinary metanephrine concentration (Supplementary Table 1).

\section{Immunohistochemistry and genotype/ phenotype correlation}

Immunohistochemistry staining of SDHB and SDHA showed lower SDHB and higher SDHA staining intensity in $S D H x$ mutated compared to SDHx wt tumors $(P=1.50 \mathrm{E}-13$ and 6.71E-06, respectively, Supplementary Fig. 2B and C). Staining intensity of SDHB (cut off $<0.504$ ) predicted the existence of $S D H x$ mutation with an area under the curve 
Table 1 A clinical characteristic of PPGL patients stratified by evidence of malignancy. Data are presented as median (IQR).

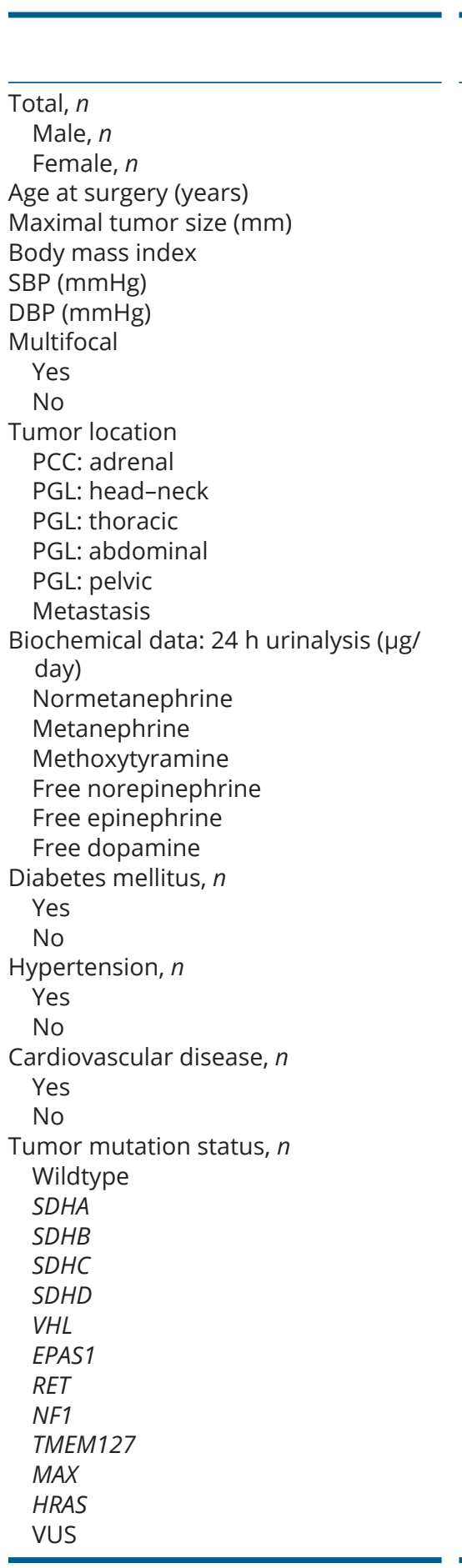

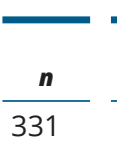

331
309
220
186
186
320

\begin{tabular}{c}
\hline Total cohort \\
\hline 153 \\
178 \\
$50(40-62)$ \\
$45(32-63)$ \\
$24.8(22.4-27.7)$ \\
$137(123-155)$ \\
$82(75-90)$ \\
36 \\
284 \\
268 \\
31 \\
5 \\
26 \\
5 \\
3
\end{tabular}

$\begin{array}{rc}178 & 2289(775-5512) \\ 189 & 617(157-2770) \\ 65 & 258(186-497) \\ 104 & 104(49-297) \\ 105 & 17(5-58) \\ 120 & 247(186-334) \\ 102 & \\ & 37 \\ 267 & 65 \\ & 216 \\ & 51\end{array}$

107

188

41
66
43
$2^{*}$
15
$5^{\dagger}$
12
21
4
36
31
2
5
8
4

\begin{tabular}{c} 
Evidence of \\
\hline Yes, $n=19$ \\
\hline 10 \\
9 \\
$51(46-64)$ \\
$73(44-88)$ \\
$24.6(22.3-27.7)$ \\
$137(132-145)$ \\
$82(75-91)$ \\
1 \\
17 \\
8 \\
1 \\
0 \\
7 \\
0 \\
-
\end{tabular}

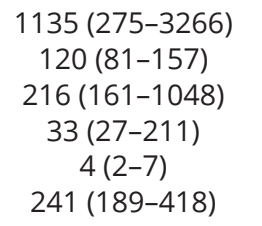

$$
\begin{gathered}
2305(805-5910) \\
650(195-2963) \\
266(190-492) \\
113(58-300) \\
20(5-63) \\
254(187-329)
\end{gathered}
$$$$
2
$$$$
5
$$$$
35
$$$$
60
$$$$
14201
$$$$
150
$$

0.003

$P$-values of less than 0.05 were considered significant and shown in bold. *One patient with SDHA and MDH2 mutation. tOne patient with promoter methylation of $S D H C$ gene.

DBP, diastolic blood pressure; PCC, pheochromocytoma; PGL, paraganglioma; SBP, systolic blood pressure; VUS, variant of unknown significance. 
of 0.928 and diagnostic sensitivity of $90.0 \%$ and specificity of $87.8 \%$ (Supplementary Fig. 2D). As expected, the low staining intensity of SDHB (cut off $<0.504$ ) was associated with malignant behavior (log-rank tests $P=0.004$, Supplementary Fig. 2E).

\section{Metabolome profiles in correlation to genotypes}

Unsupervised hierarchical clustering analysis of the MALDI-MSI dataset from 184 genetically annotated tumors revealed three groups with distinct metabolic pattern (Supplementary Fig. 3A); group a containing 19 cluster 1 tumors (76.0\%; SDHx, VHL and EPAS1 mutation), group b with 34 wt tumors (26.2\%), and group c with 21 cluster 2 tumors (72.4\%; RET, NF1, TMEM127, MAX and HRAS mutation). An analysis of 144 tumors that belonged to cluster 1 and cluster 2 was performed by heat map based cluster analysis and ortho-PLSDA (Fig. 1A and B). Thereby, three groups were identified with group $\mathrm{A}$, in which 23 tumors (74.2\%) were from cluster 1, group B, in which an equal number of cluster 1 and 2 tumors were included, and group C, in which 45 tumors from cluster $2(86.5 \%)$ were included (Fig. 1A). While overlap and continuous changes were evident between cluster 1 and 2 PPGLs, among approximately $2000 \mathrm{~m} / \mathrm{z}$ species over a mass range of $50-1000 \mathrm{~m} / \mathrm{z}, 266$ metabolites were found to be significantly different (more than two-fold change and $P<0.05$ ) between the two clusters. Expectedly, these included metabolites related to catecholamine synthesis, such as L-DOPA and 3-methoxytyramine sulfate, which were more abundant in cluster $2(P=6.42 \mathrm{E}-06$ and 1.40E-05, respectively, Fig. 1C) and norepinephrine with higher abundance in cluster 1 ( $P=0.023$, Fig. $1 C)$.

To take into account a potential bias based on sample age, differences in sample fixation and specimen preparation, we assigned age and center of individual samples to PLSDA. Notably, and at variance to the genetic status, neither sample age nor place of origin did significantly impact on the attribution of the individual sample data to the cluster1 or 2 (Supplementary Fig. 3B).

Pathway enrichment analysis of significantly different metabolites between cluster 1 and 2 revealed altered pathways such as 'purine metabolism', 'tryptophan metabolism', 'pyruvate metabolism', 'inositol metabolism', among others (Supplementary Table 2A). Metabolites related to purine metabolism, AMP, ADP and ATP, were found to be more abundant in cluster $2(P=2.64 \mathrm{E}-04$, 0.022 and 0.002 , respectively, Fig. 1D). Further clusterrestricted examination revealed additional differences in purine metabolism with a higher adenine nucleoside phosphate content in VHL mutated tumors in comparison to $S D H x$ mutated tumors (Supplementary Fig. 3C). In addition, metabolites related to tryptophan metabolism, including xanthurenic acid and 8-methoxykynurenate, were found to be significantly more abundant in cluster 2 tumors $(P=2.35 \mathrm{E}-09$ and $5.06 \mathrm{E}-11$, respectively, Fig. $1 \mathrm{E}$ and Supplementary Table 2A). As both metabolites are part of the kynurenine pathway, further members of this pathway were analyzed more closely and kynurenic acid and 7,8-dihydroxykynurenic acid were more abundant in cluster 2 , although with a less than two-fold difference (fold change $=1.948$ and $1.890, P=3.07 \mathrm{E}-06$ and $1.01 \mathrm{E}-05$, respectively).

\section{Comparison of metabolic profiles between tumor and in vitro samples}

In order to provide further insights into the pathogenic mechanisms of PPGLs, we compared the metabolic profiles between in situ samples and in vitro cell models. siRNA knockdown of $S d h b$ and $V h l$ genes was introduced in PC12 cells (Supplementary Fig. 4A and B). Thereby, we found that knockdown of $S d h b$ expression in PC12 cells led to the accumulation of succinate and a higher succinate/fumarate ratio (Supplementary Fig. 4C) as expected for cluster 1 tumors. Considering the fact that PC12 cells are lacking a functional Max gene $(25,26)$, cells transfected with scrambled siRNA were expected to represent a cluster 2-like status. Using these in vitro models, 198 and 227 metabolites were identified as differentially regulated $(P<0.05)$ in $S d h b$ and $V h l$ knockdown cells in comparison to cells transfected with scrambled siRNA, respectively. Thirtytwo metabolites such as tryptophan $(P=0.001$ and 0.014 , respectively, Supplementary Fig. 4D) were commonly more abundant in $S d h b$ and $V h l$ knockdown cells. Seventyfive metabolites were commonly decreased in $S d h b$ and Vhl knockdown cells. Based on pathway enrichment analysis, these were classified into 'purine metabolism', 'amino sugar metabolism' and 'fatty acid biosynthesis' (Supplementary Table 2B). As pathway enrichment analysis indicated, metabolites of 'purine' and 'pyrimidine metabolism' in human PPGLs to be decreased in cluster 1 (Supplementary Table 2A and Fig. 2A), we focused on nucleoside-related metabolites in the PC12 cell models. In $S d h b$ and $V h l$ knockdown cells we observed decreased amounts of inosine (both $P=0.006$ vs scrambled siRNA, Fig. $2 \mathrm{~A}$ and $\mathrm{B}$ ) and uridine (both $P=0.002$, Fig. $2 \mathrm{~A}$ and $\mathrm{B}$ ). Compounds related to inositol metabolism were decreased in cluster 1 (Supplementary Table 2A and Fig. 2A), and accordingly, inositol cyclic phosphate as inositol-related 

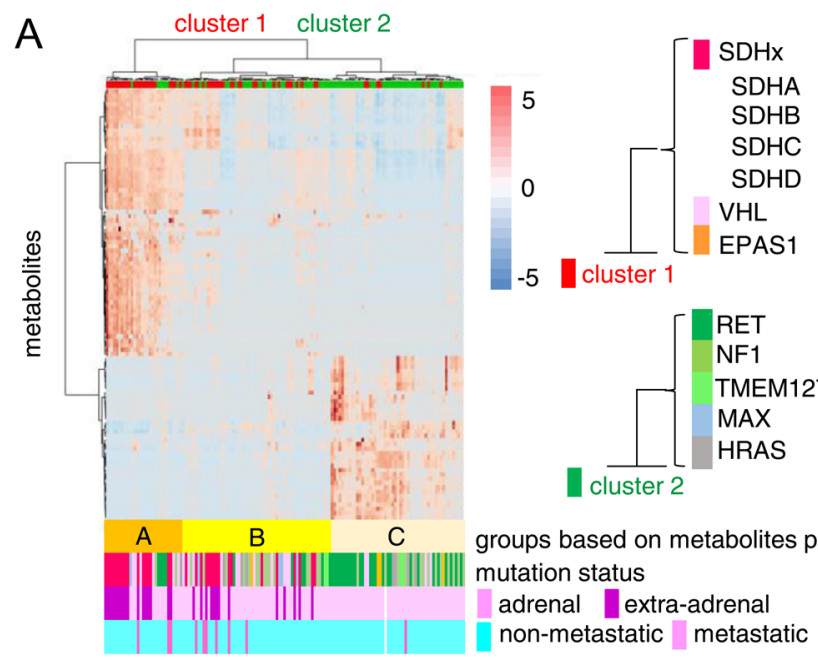

groups based on metabolites pattern

mutation status

adrenal extra-adrenal

non-metastatic metastatic

\section{B}

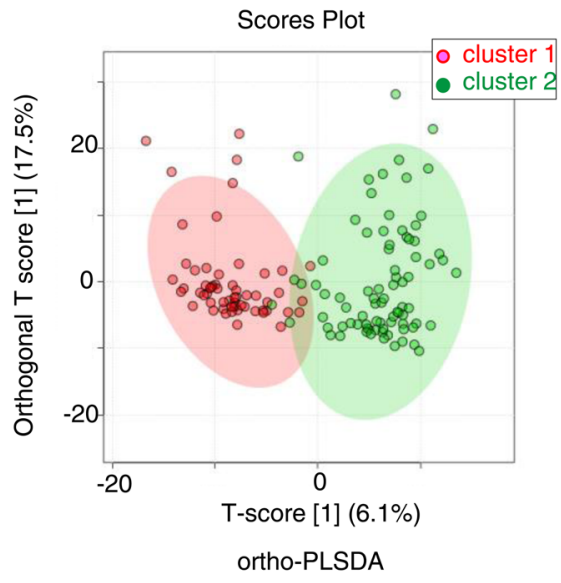

C

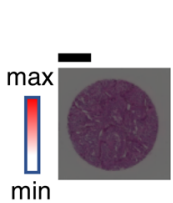

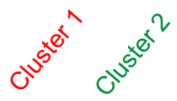

L-Dopa

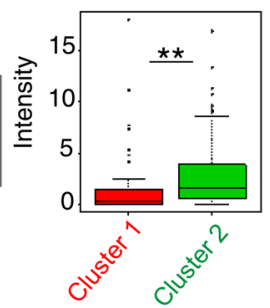

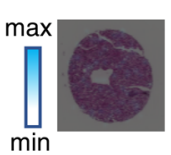

$c^{5^{e^{2}}} u^{5^{e^{x^{2}}}}$
3-Methoxytyramine sulfate

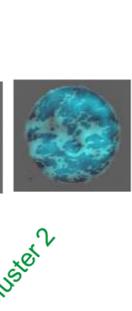

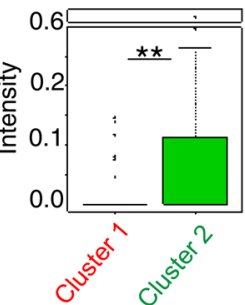

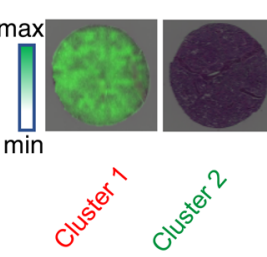

Norepinephrine

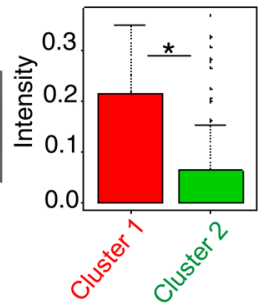

Purine metabolism
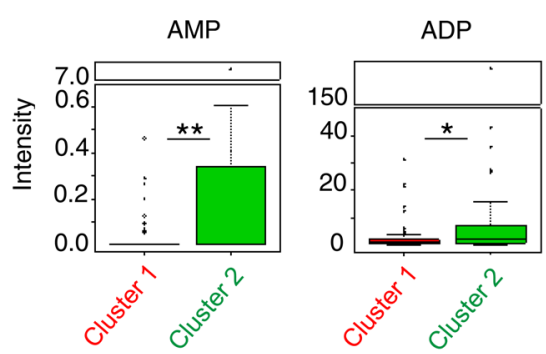

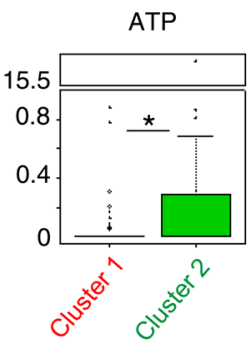

E

Tryptophan metabolism

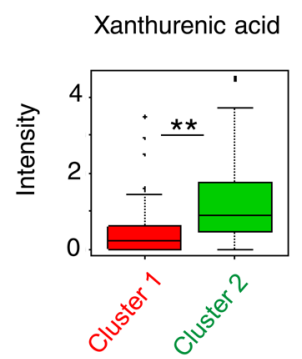

8-Methoxykynurenate

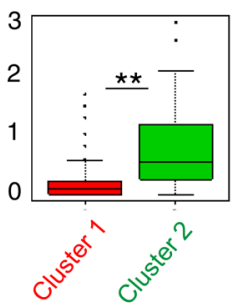

\section{Figure 1}

Tissue metabolomic profiles of PPGLs. Unsupervised hierarchical clustering analysis (A) and orthogonal partial least squares discriminant analysis (Ortho-PLSDA, B) of metabolome profiles of 141 PPGLs separates cluster 1 and 2. Groups based on metabolite pattern, mutation status, tumor location and metastatic behavior are shown below the heat map. Distribution of patient numbers with regard to mutation status is shown. Intensities of the catecholamine metabolites L-dopa,

3-methoxytyramine sulfate, and norepinephrine, in clusters 1 and 2 and representative images are shown in (C). Significant differences of purine metabolites AMP, ADP and ATP are depicted in (D), and of tryptophan metabolites xanthurenic acid and 8-methoxykynurenate in (E). Mann-Whitney $U$ test and Kruskal-Wallis test were used for statistical analysis. ${ }^{*} P<0.05,{ }^{*} P<$ 0.001. Scale bars: $500 \mu \mathrm{m}$. 
A

\begin{tabular}{|c|c|c|c|}
\hline & $\begin{array}{l}\text { Human PP } \\
\text { cluster } 1 \text { vs cl}\end{array}$ & & \\
\hline & & $\begin{array}{l}\text { Fold } \\
\text { change }\end{array}$ & $P$-value \\
\hline Metabolites cont & cleoside & $\begin{array}{l}\text { cluster } 1 \\
\text { /cluster } 2\end{array}$ & \\
\hline & AMP & 0.091 & 2.64E-04 \\
\hline & ATP & 0.192 & 0.002 \\
\hline & ADP & 0.257 & 0.022 \\
\hline & GTP & 0.270 & 4.25E-04 \\
\hline & 7-Methyl-GDP & 0.320 & 0.003 \\
\hline Purine & Adenylsuccinate & 0.384 & 0.003 \\
\hline वाँाए & GDP & 0.387 & 3.48E-05 \\
\hline & dGTP & 0.408 & 0.007 \\
\hline & Cyclic AMP & 0.438 & 0.024 \\
\hline & dAMP & 0.439 & 0.019 \\
\hline & Cyclic GMP & 0.470 & 0.007 \\
\hline & 1-Methyladenosine & 0.492 & 0.001 \\
\hline & 3'-UMP & 0.327 & 3.75E-04 \\
\hline Pyrimidine & UDP & 0.338 & 0.002 \\
\hline 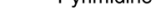 & dCTP & 0.418 & 1.31E-05 \\
\hline
\end{tabular}

Inositol metabolism

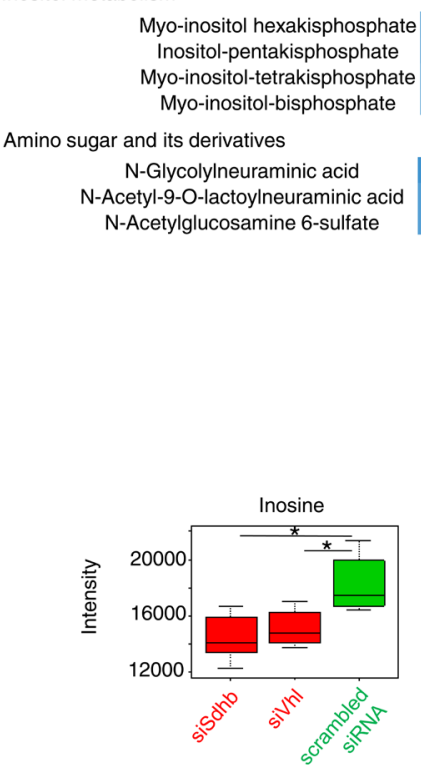

$\mathrm{N}$-Acetylneuraminic acid

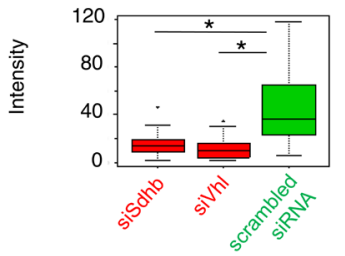

B

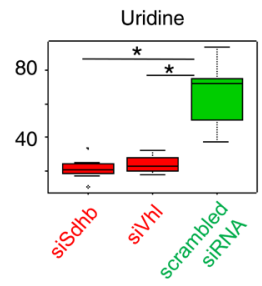

Glucosamine

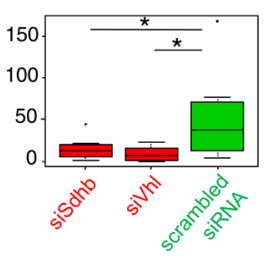

PC12 cells

cluster 1 -like status vs cluster 2 -like statu

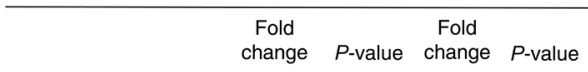

sisdhb sivhl

/scrambled siRNA /scrambled siRNA

\begin{tabular}{|l|l|l|l|}
\hline 0.025 & 0.222 & 0.011 & 0.107 \\
0.442 & 0.002 & 0.703 & 0.019
\end{tabular}

$\begin{array}{lllll}0.442 & 0.002 & 0.703 & 0.019\end{array}$

\begin{tabular}{l|l|l|l|}
0.490 & 0.002 & 0.803 & 0.171 \\
\hline
\end{tabular}

\begin{tabular}{|l|l|l|l|}
\hline 0.551 & 0.011 & 0.562 & 0.030 \\
\hline
\end{tabular}

Guanosine

Hypoxanthin

S-(5'-Adenosyl)-L-

homocysteine

\begin{tabular}{l|l|l|l|}
0.579 & $5.98 \mathrm{E}-07$ & 0.785 & 0.022 \\
\hline
\end{tabular}

\begin{tabular}{|l|l|l|l|l|l|}
\hline MP sodium salt & 0.608 & 0.200 & 1.237 & 0.714 \\
\hline
\end{tabular}

\begin{tabular}{|l|l|l|l|}
0.789 & 0.006 & 0.789 & 0.006 \\
\hline
\end{tabular}

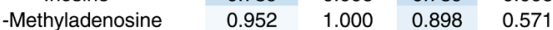

\begin{tabular}{|l|l|l|l|l|}
\hline Methylguanosine & 1.454 & 0.008 & 0.756 & 0.141 \\
\hline
\end{tabular}

\begin{tabular}{l|l|l|l|l|l|}
\hline Adenosine & 1.647 & 0.001 & 0.637 & 0.002 \\
\hline
\end{tabular}

$\begin{array}{llllll}\text { Deoxyinosine } & 1.777 & 0.045 & 1.561 & 0.284\end{array}$

\begin{tabular}{l|l|l|l|l|l} 
Cyclic AMP & 1.808 & $4.84 \mathrm{E}-06$ & 1.506 & $5.69 \mathrm{E}-04$
\end{tabular}

DP-D-Glucose

Uridine

Cytidine

UDP-D-

Glucose, disodium salt

CDP

\begin{tabular}{|l|l|l|l|}
\hline 0.004 & 0.608 & 0.003 & 0.106 \\
\hline 0.328 & 0.002 & 0.364 & 0.002 \\
\hline 0.653 & 0.185 & 0.833 & 0.768 \\
\hline
\end{tabular}

$0.267 \quad 0.611$

\begin{tabular}{l|l|l|l|}
\hline 1.282 & 0.036 & 1.620 & 0.500 \\
\hline 1.519 & 0.021 & 1.279 & 0.191
\end{tabular}

$\begin{array}{llll}1.519 & 0.021 & 1.279 & 0.191\end{array}$

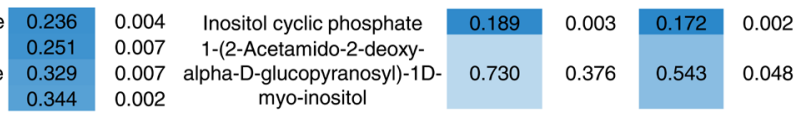

\section{$\begin{array}{ccc}0.249 & 0.002 & \text { Glucosamine } \\ 0.337 & 1.65 \mathrm{E}-05 & \text { N-Acetylneuraminic acid } \\ 0.341 & 1.53 \mathrm{E}-09 & \text { Lactosamine }\end{array}$} Chitobiose

$\mathrm{N}-$ Acetyl-D-gl

\begin{tabular}{|c|c|c|c}
0.297 & 0.012 & 0.197 & 0.002 \\
0.327 & 0.001 & 0.265 & $4.19 \mathrm{E}-04$ \\
\hline .328 & $2.69 \mathrm{E}-04$ & 0.211 & $5.06 \mathrm{E}-05$
\end{tabular}

$\begin{array}{lllll}0.328 & 2.69 \mathrm{E}-04 & 0.211 & 5.06 \mathrm{E}-05\end{array}$

\begin{tabular}{lllll}
0.696 & 0.127 & 0.663 & 0.093 \\
\hline
\end{tabular}

\begin{tabular}{|l|l|l|l|l|}
\hline & 0.781 & 0.114 & 0.621 & 0.005 \\
\hline
\end{tabular}

\begin{tabular}{|l|l|l|l|l|l|}
\hline cetamido-2-deoxy-beta-D- & 0.834 & 0.437 & 0.843 & 0.487 \\
\hline
\end{tabular}

glucosylamine

ridine 5 -diphospho-N-

etylgalactosamin

$\begin{array}{llll}1.078 & 0.769 & 1.018 & 0.943\end{array}$

N-Acetyl-D-galactosamin

1.258

$\begin{array}{lll}1.000 & 1.042 \quad 1.000\end{array}$
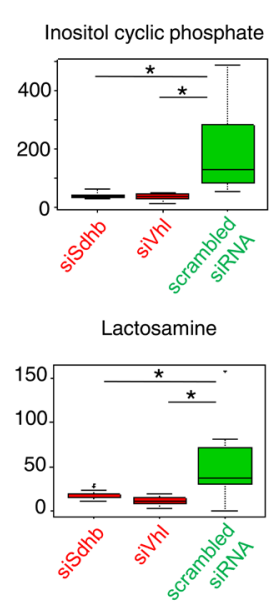

\section{Figure 2}

Comparison of metabolomic features between PPGLs and PC12 cells. Comparison of metabolites included in 'metabolites containing nucleoside', 'inositol metabolism' and 'amino sugar and its derivatives' with regard to 'cluster 1 vs cluster 2 ' in human PPGLs and 'siSdhb or siVh/ vs scrambled siRNA' in PC12 cells (A). Significant differences inosine, uridine, inositol cyclic phosphate, $\mathrm{N}$-acetylneuraminic acid, glucosamine, and lactosamine in PC12 cells were observed (B). Mann-Whitney $U$ test and Kruskal-Wallis test were used for statistical analysis. $* P<0.05$. 


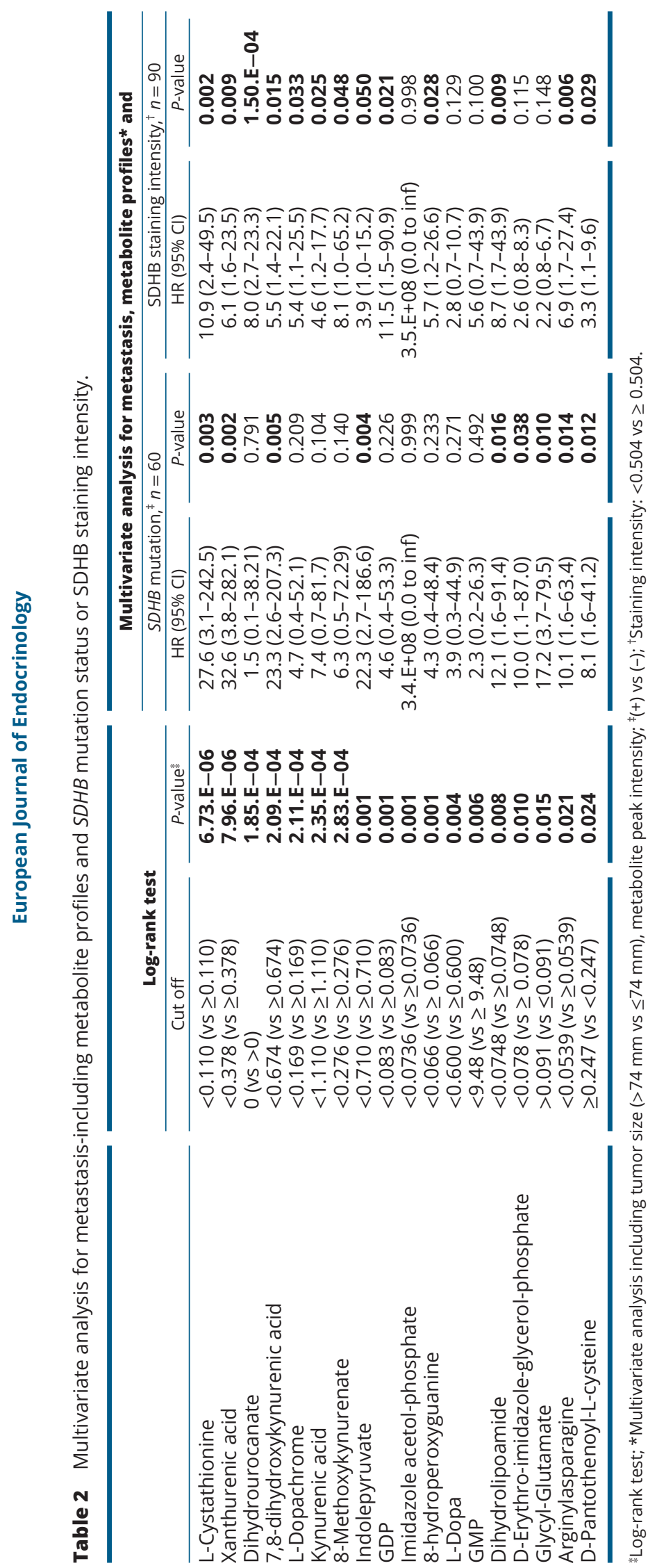

metabolite was also decreased in $S d h b$ and $V h l$ knockdown PC12 cells ( $P=0.003$ and 0.002, Fig. 2A and B). Decreased abundance of $\mathrm{N}$-acetylglucosamine 6-sulfate and sialic acids such as $\mathrm{N}$-glycolylneuraminic acid and $\mathrm{N}$-acetyl-9-Olactoylneuraminic acid were observed in cluster 1 human tumors $(P=1.53 \mathrm{E}-09,0.002$ and $1.65 \mathrm{E}-05$, respectively, Fig. 2A). In $S d h b$ and $V h l$ knockdown cells we found less aminosugars $N$-acetylneuraminic acid $(P=0.001$ and 4.19E-04, respectively), glucosamine $(P=0.012$ and 0.002 , respectively), and lactosamine $(P=2.69 \mathrm{E}-04$ and 5.06E-05, respectively, Fig. 2A and B).

\section{Impact of metabolic profiles on the diagnosis of metastatic PPGLs}

Tissue metabolomic parameters were further correlated with clinical annotation of malignancy. Because clinical exclusion of malignant PPGL is dependent on an appropriate follow-up period, non-metastatic PPGL was defined as the absence of metastasis after a follow-up of more than 5 years. Based on this cohort of 35 patients (Supplementary Table 3), clustering analysis revealed distinct metabolite profiles between malignant and apparently benign PPGLs (Fig. 3A), with 154 metabolites being significantly different (with a more than two-fold change and $P<0.05$ ) between the groups. We further assessed occurrence of metastasis with the Kaplan-Meier method and log-rank test using optimized cutoffs for metabolite intensity. Forty-eight metabolites (of which 18 could be annotated) in primary tumors were significantly associated with metastasis-free survival (Table 2). Metabolites implicated in the kynurenine pathway such as xanthurenic acid (Fig. 3B), 7,8-dihydroxykynurenic acid, kynurenic acid and 8-methoxykynurenate were among the metabolites with a predictive value (log-rank tests $P=7.96 \mathrm{E}-06,2.09 \mathrm{E}-04,2.35 \mathrm{E}-04$, and 2.83E-04, respectively, Table 2). The number of kynurenine pathway metabolites with lower than cut-off intensity was associated with shorter metastasis-free survival (logrank tests $P=1.66 \mathrm{E}-05$, hazard ratio, 3.61, $P=2.33 \mathrm{E}-04$, Fig. 3C). Thereby, PPGLs enriched in metabolites of the kynurenine pathway were found to be associated with a more favorable biological behavior. Multivariate analysis, including the intensity of metabolite, maximal tumor size (>74 $\mathrm{mm}$ vs $\leq 74 \mathrm{~mm}$ ) and $S D H B$ mutation status (mutated vs wild type), revealed nine metabolites as independent factors for metastasis-free survival (Table 2). By using SDHB staining intensity $(<0.504$ vs $\geq 0.504)$ in lieu of mutation status, multivariate analysis including the intensity of metabolite and maximal tumor size retained 
13 metabolites as independent factors for metastasis-free survival. Seven metabolites, including xanthurenic acid and 7,8-dihydroxykynurenic acid from the kynurenine pathway, were overlapping for both $S D H B$ mutation status and SDHB staining intensity. In addition, low xanthurenic acid levels predicted the existence of metastasis (AUC $=0.809,95 \% \mathrm{CI}=0.654-0.964, P=9.59 \mathrm{E}-05)$ with higher accuracy than $S D H B$ mutation status $(A U C=0.772$, 95\% $\mathrm{CI}=0.611-0.933, \quad P=9.44 \mathrm{E}-04)$ and maximum tumor size $(\mathrm{AUC}=0.599,95 \% \mathrm{CI}=0.366-0.831, P=0.405$, Fig. 3D) alone. To combine the intensity of metabolites, maximal tumor size and $S D H B$ mutation status or - as an alternative - SDHB staining intensity, a scoring system was established to assess the risk of metastasis. Using the low intensity of xanthurenic acid $(<0.378)$, larger tumor size (>74 mm), SDHB mutation (mutated), the sum of these risk factors could discriminate metastatic from non-metastatic PPGLs (hazard ratio, 5.427; $P=3.46 \mathrm{E}-05$, Fig. 3E). From 60 patients, eight were diagnosed with metastatic disease. Seven of those $(88 \%)$ had low xanthurenic acid tissue intensity. On the other hand, only five patients (63\%) developed metastasis in the context of $S D H B$ mutation. Thus, two additional patients with metastasis could be identified by using low xanthurenic acid tissue intensity as a marker of malignant behavior (Supplementary Fig. $5 \mathrm{~A})$. When applying lower SDHB staining intensity $(<0.504)$ instead of $S D H B$ mutation status (mutated) as a risk factor, the scoring system remained discriminative for PPGLs with metastasis (hazard ratio, 4.818; $P=1.30 \mathrm{E}-06$, Supplementary Fig. 5B). Overall, a low abundance of metabolites in the tryptophan and kynurenine pathways were found to be associated with metastatic disease and in the context of cluster 1 mutations (Fig. 3F).

\section{Discussion}

Herein, we report an in-depth analysis of tissue metabolome profiles based on MALDI-mass spectrometry imaging in a large cohort of PPGL patients. Based on the current untargeted metabolomic profiles, we provide insight in the specific metabolic alterations related to the genetic events present in cluster 1 and cluster 2 tumors. We still found a group of tumors with an intermediate metabolic phenotype of more gradual changes in some metabolites leading to overlap between the genetic clusters.

A previous study had demonstrated a higher succinate/ fumarate ratio, a well-known hallmark of SDHx mutated tumors, in FFPE samples, by applying ultrahigh-pressure liquid chromatography with tandem mass spectrometry
(15). Based on the current untargeted metabolomic profiles, we further found contrasting patterns between tumors of cluster 1 and cluster 2, although gradual changes of some metabolites with overlap between the clusters were present as well. Metabolites related to 'purine metabolism' including AMP, ADP and ATP were present at lower levels in cluster 1 tumors, consistent with findings from previous studies using proton NMR $(16,17)$. Disruption of respiratory enzyme complex II in $S D H x$ mutated tumors is likely to reduce tumor content of AMP/ADP/ATP. Previous studies have indicated increased glycolysis preferentially in VHL mutated tumors (27) and higher accumulation of AMP/ADP/ATP in VHL in comparison to SDHx mutated tumors $(28,29)$. Our observation based on MALDI imaging corroborates these reports in terms of ADP distribution in SDHx and VHL mutated tumors.

It has been shown that lower tissue catecholamine content is a characteristic of tumors from cluster 1 (30). Our observation confirmed lower contents of L-dopa and 3-methoxytyramine sulfate as catecholamine-related metabolites in cluster 1 . The presence of the sulfated metabolite of dopamine in cluster 2 was unexpected because it had not been reported previously. In fact, all previous analyses of tumor metabolites were targeted approaches, for example, focusing on Krebs cycle metabolites or polyamines. Canonically, sulfation of 3-methoxytyramin is considered to occur mostly in the liver. However, interrogation of TCGA data demonstrated that a variety of sulfotransferases is expressed at different levels in PPGL (data not shown) and might contribute to the sulfation of 3-methoxytyramine. Although the previous study had demonstrated, that cluster 2 tumors contained more norepinephrine and epinephrine than those of cluster 1, the higher amounts of norepinephrine in cluster 1 tumors observed in the present study could well be related to a lack of PNMT gene expression, which is a representative feature of cluster 1 PPGLs (30). While it is possible that the stability of catecholamines in FFPE tissue might have affected the measurement of norepinephrine, generally, the tissue metabolome profiles did not depend on sample age and center distribution (Supplementary Fig. 3B).

We further used PC12 cells, which lack a functional Max gene that results in a cluster 2-like status. By knockdown of $S d h b$ and $V h l$, we implemented a model of cluster 1-like status. Lower contents of $\mathrm{N}$-acetylneuraminic acid in PC12 cells and $\mathrm{N}$-acetyl-9-O-lactoylneuraminic acid and $N$-glycolylneuraminic acid in human PPGLs were evident in cluster 1 (-like status). There is a growing body of evidence demonstrating that cancer cells have significantly 
A

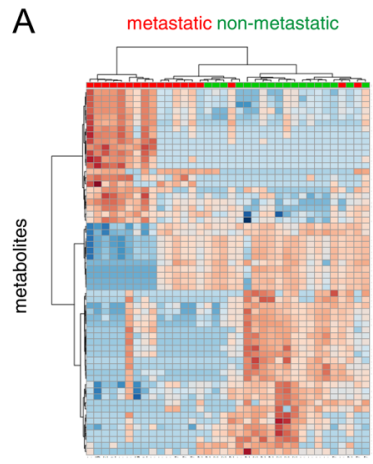

unguided hierarchical cluster analysis

D

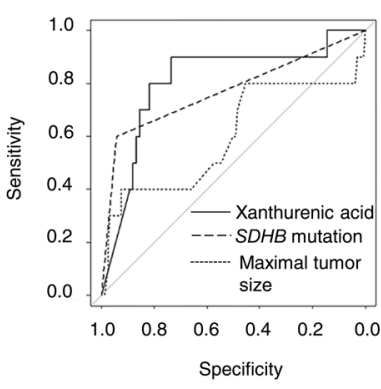

B

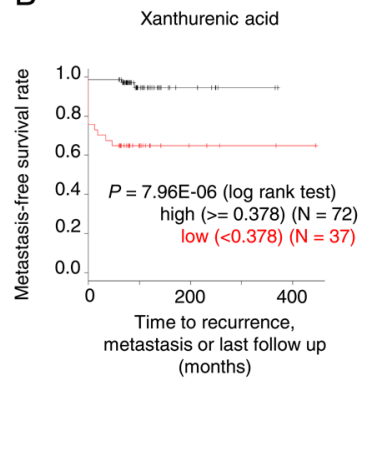

E

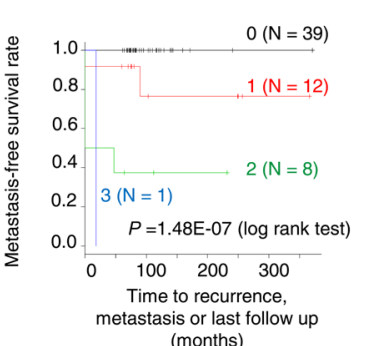

C

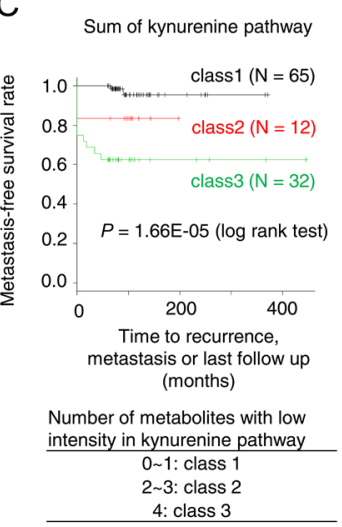

$\mathrm{F}$

\begin{tabular}{lccc} 
& hazard ratio & $95 \% \mathrm{Cl}$ & $P$-value \\
\hline Sum of risk factors ( per 1) & 5.427 & $(2.448-12.23)$ & $3.46 \mathrm{E}-05$
\end{tabular}

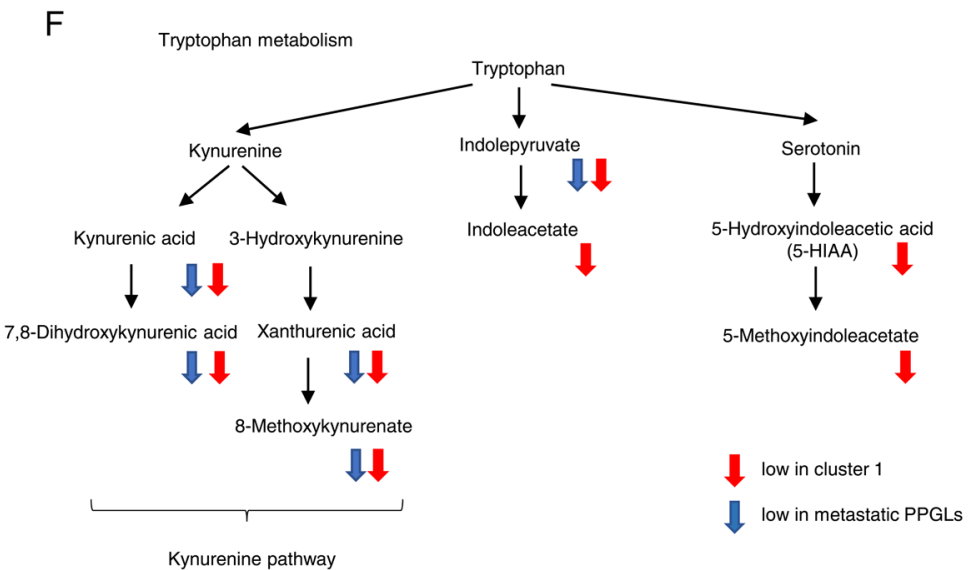

Figure 3

Tissue metabolome characteristics of metastatic PPGLs. Unsupervised hierarchical clustering analysis of metabolome profiles of 36 metastatic and non-metastatic PPGLs with long term follow-up are provided in (A). Kaplan-Meier plots for metastasis-free survival in relation to high and low xanthurenic acid (B). Kaplan-Meier plots for metastasis-free survival based on the number of metabolites with low intensity in kynurenine pathway xanthurenic acid $(<0.378)$, 7,8-dihydroxykynurenic acid $(<0.674)$, kynurenic acid $(<1.110)$ and 8-methoxykynurenate $(<0.276)(C)$. ROC curves for prediction of metastatic behavior by xanthurenic acid, SDHB mutation status and maximal tumor size and area under curves (AUC) are provided (D). Kaplan-Meier plots for metastasis-free survival in relation to a scoring system using maximal tumor size, SDHB mutation status and xanthurenic acid (E). Kaplan-Meier plots for metastasis-free survival in relation to the scoring system and the examination of the scoring system by cox proportional hazards model are shown. Log-rank test was used to statistically compare the curves and $P$-values are provided. Schematic diagram of tryptophan metabolism (F). Metabolites with low intensity in cluster 1 in comparison to cluster 2 are indicated as red arrows. Metabolites with low intensity and associated with metastatic PPGLs are indicated as blue arrows. 
elevated levels of sialic acid (31). Although detailed mechanisms between the genotype and the content of sialic acids in PC12 cells and PPGLs are to be elucidated, it is interesting to note this is a common molecular fingerprint of cluster 1 (-like status) in human PPGLs and PC12 cells that underscores its potential functional relevance.

Discrimination of metabolome profiles with respect to malignant and apparently benign PPGLs identified 18 metabolites, which were associated with metastasis-free survival. Nine of these metabolites were significantly associated with malignant potential independently of clinical features and $S D H B$ mutation status. Thereby, our findings add to molecular markers that earlier studies had identified, such as telomerase activation, $A T R X$ gene mutations and specific miRNA signatures to predict the existence of metastatic PPGLs $(32,33)$.

The significant correlation between metastatic spread and kynurenine pathway metabolites sheds light on potential disease mechanisms. We observed low intensity of tryptophan-related metabolites in cluster 1 tumors, while metabolites related to serotonin such as 5-HIAA and 5-methoxyindoleacetate were not associated with metastatic behavior. Conversely, metabolites from the kynurenine pathway were less abundant in cluster 1 tumors and further associated with metastatic behavior. The kynurenine pathway is the principal route of tryptophan catabolism. Overproduction of kynurenine from tryptophan, which is an initial step of the kynurenine pathway and promoted by overactivation of indoleamine 2,3-dioxygenase (IDO), is known to predict poor prognosis of several cancers (34). While kynurenine was not among the differentially distributed metabolites between benign and malignant PPGLs in our analysis, kynurenic acid and xanthurenic acid were detected at lower intensities in malignant PPGLs. It is conceivable that - beyond the immunological role of the kynurenine pathway kynurenic acid itself may have anti-proliferative activity similar to what has been reported for colon cancer and renal cancer cell lines $(35,36)$.

There are some limitations of the current study that need to be considered: distribution of metabolites in FFPE tissues, which were used for the MALDI imaging analysis, are likely affected by fixation and embedding procedures. Despite this potential bias, our observation of decreased nucleoside phosphates in cluster 1 tumors is consistent with previous studies and confirms the validity of our analysis. In addition, we recover catecholamines and their metabolites as positive controls. While we identified metabolic pathways associated with the malignant potential of PPGLs, metastasis-free survival rate, could only be calculated for 109 from 344 tumors because of the required long follow-up. Similarly, multivariate analysis, which required tumor size, mutation status and metabolite data, was restricted to only 60 tumors because of missing data mainly on the tumor mutation status. In addition, as PPGLs can recur or metastasize very late in the course of the disease any restricted observation period includes limitations in the grouping of apparently benign tumors. The applied observation period of five years represents a compromise that balances a clinically meaningful time frame with the availability and age of samples and the resulting group size. Finally, considering the exploratory character of our study, confirmatory tests should be performed in the future. While it will be difficult to implement a similarly large study using FFPE tissues, more focused metabolomic approaches on prospectively collected fresh frozen tissue and in experimental model systems would be useful to validate and extend our findings.

In summary, the present study indicates distinct metabolomic profiles of PPGLs with regard to genotype classification between clusters 1 and 2. Comparison of metabolome between PPGLs and in vitro model showed similarities in inositol metabolism and the differential expression of sialic acid between clusters 1 and 2. The association of low kynurenine pathway activity in metastatic PPGL is novel and provides unprecedented insights into the pathophysiology of PPGLs.

\section{Supplementary materials}

This is linked to the online version of the paper at https://doi.org/10.1530/ EJE-20-1407.

\section{Declaration of interest}

Martin Fassnacht is on the editorial board of EJE. Martin Fassnacht was not involved in the review or editorial process for this paper, on which he is listed as an author. All the other authors declare no conflicts of interest.

\section{Funding}

This study was supported by the German Research Foundation (DFG) project number 314061271 (CRC/TRR 205) to F.B., M. Reincke., M.F., A.K.W., N.B., M.P. and P.W., and INST 515/28-1 FUGG to M.P., and the German Cancer Aid (project number 70112617) to F.B., M.F., M.K. and A.K.W., and by the Clinical Research Priority Program of the University of Zurich for the CRPP HYRENE to F.B. M. Robledo was supported by the Instituto de Salud Carlos III (ISCIII), Acción Estratégica en Salud, (grant number PI17/01796), and the Paradifference Foundation. M.M. was supported by the Japan Heart Foundation/Bayer Yakuhin Research Grant Abroad and a postdoctoral fellowship of the Uehara Memorial Foundation. This study is partially based on the FP7 funded ENSAT-Cancer program. 


\section{Author contribution statement}

Masanori Murakami, Na Sun, Matthias Kroiss, Felix Beuschlein contributed equally to this work.

\section{Acknowledgements}

The authors would like to thank Ulrike Buchholz, Claudia-Mareike Pflüger, Andreas Voss, Jana Drechsler, Brigitte Mauracher, Petra Rank, Christina Brugger and Kerstin Schaefer for excellent technical assistance. The authors also thank Professor Natalia Pellegata for kindly providing PC12 cells.

\section{References}

1 Lenders JW, Duh QY, Eisenhofer G, Gimenez-Roqueplo AP, Grebe SK, Murad MH, Naruse M, Pacak K, Young WF Jr \& Endocrine Society. Pheochromocytoma and paraganglioma: an endocrine society clinical practice guideline. Journal of Clinical Endocrinology and Metabolism 201499 1915-1942. (https://doi.org/10.1210/jc.20141498)

2 Buffet A, Morin A, Castro-Vega LJ, Habarou F, Lussey-Lepoutre C, Letouze E, Lefebvre H, Guilhem I, Haissaguerre M, Raingeard I et al. Germline mutations in the mitochondrial 2-oxoglutarate/ malate carrier SLC25A11 gene confer a predisposition to metastatic paragangliomas. Cancer Research 201878 1914-1922. (https://doi. org/10.1158/0008-5472.CAN-17-2463)

3 Favier J, Amar L \& Gimenez-Roqueplo AP. Paraganglioma and phaeochromocytoma: from genetics to personalized medicine. Nature Reviews: Endocrinology 201511 101-111. (https://doi.org/10.1038/ nrendo.2014.188)

4 Dahia PL. Pheochromocytoma and paraganglioma pathogenesis: Learning from genetic heterogeneity. Nature Reviews: Cancer 201414 108-119. (https://doi.org/10.1038/nrc3648)

5 Fishbein L, Leshchiner I, Walter V, Danilova L, Robertson AG, Johnson AR, Lichtenberg TM, Murray BA, Ghayee HK, Else T et al. Comprehensive molecular characterization of pheochromocytoma and paraganglioma. Cancer Cell 201731 181-193. (https://doi. org/10.1016/j.ccell.2017.01.001)

6 Dahia PL, Ross KN, Wright ME, Hayashida CY, Santagata S, Barontini M, Kung AL, Sanso G, Powers JF, Tischler AS et al. A HIF1alpha regulatory loop links hypoxia and mitochondrial signals in pheochromocytomas. PLoS Genetics 20051 72-80. (https://doi. org/10.1371/journal.pgen.0010008)

7 Flynn A, Dwight T, Harris J, Benn D, Zhou L, Hogg A, Catchpoole D, James P, Duncan EL, Trainer A et al. Pheo-type: a diagnostic geneexpression assay for the classification of pheochromocytoma and paraganglioma. Journal of Clinical Endocrinology and Metabolism 2016 101 1034-1043. (https://doi.org/10.1210/jc.2015-3889)

8 Castro-Vega LJ, Buffet A, De Cubas AA, Cascon A, Menara M, Khalifa E, Amar L, Azriel S, Bourdeau I, Chabre O et al. Germline mutations in FH confer predisposition to malignant pheochromocytomas and paragangliomas. Human Molecular Genetics 201423 2440-2446. (https://doi.org/10.1093/hmg/ddt639)

9 Amar L, Bertherat J, Baudin E, Ajzenberg C, Bressac-de Paillerets B, Chabre O, Chamontin B, Delemer B, Giraud S, Murat A et al. Genetic testing in pheochromocytoma or functional paraganglioma. Journal of Clinical Oncology 200523 8812-8818. (https://doi.org/10.1200/ JCO.2005.03.1484)

10 Amar L, Baudin E, Burnichon N, Peyrard S, Silvera S, Bertherat J, Bertagna X, Schlumberger M, Jeunemaitre X, Gimenez-Roqueplo AP et al. Succinate dehydrogenase $B$ gene mutations predict survival in patients with malignant pheochromocytomas or paragangliomas. Journal of Clinical Endocrinology and Metabolism 200792 3822-3828. (https://doi.org/10.1210/jc.2007-0709)
11 Ayala-Ramirez M, Feng L, Johnson MM, Ejaz S, Habra MA, Rich T, Busaidy N, Cote GJ, Perrier N, Phan A et al. Clinical risk factors for malignancy and overall survival in patients with pheochromocytomas and sympathetic paragangliomas: primary tumor size and primary tumor location as prognostic indicators. Journal of Clinical Endocrinology and Metabolism 201196 717-725. (https://doi. org/10.1210/jc.2010-1946)

12 Eisenhofer G, Pacak K, Huynh TT, Qin N, Bratslavsky G, Linehan WM, Mannelli M, Friberg P, Grebe SK, Timmers HJ et al. Catecholamine metabolomic and secretory phenotypes in phaeochromocytoma. Endocrine-Related Cancer 201118 97-111. (https://doi.org/10.1677/ERC10-0211)

13 Eisenhofer G, Huynh TT, Elkahloun A, Morris JC, Bratslavsky G, Linehan WM, Zhuang Z, Balgley BM, Lee CS, Mannelli M et al. Differential expression of the regulated catecholamine secretory pathway in different hereditary forms of pheochromocytoma. American Journal of Physiology: Endocrinology and Metabolism 2008295 E1223-E1233. (https://doi.org/10.1152/ajpendo.90591.2008)

14 Erlic Z, Kurlbaum M, Deutschbein T, Nolting S, Prejbisz A, Timmers HJ, Richter S, Prehn C, Weismann D, Adamski J et al. Metabolic impact of pheochromocytoma/paraganglioma: Targeted metabolomics in patients before and after tumor removal. European Journal of Endocrinology 2019181 647-657. (https://doi.org/10.1530/EJE-19-0589)

15 Richter S, Peitzsch M, Rapizzi E, Lenders JW, Qin N, de Cubas AA, Schiavi F, Rao JU, Beuschlein F, Quinkler M et al. Krebs cycle metabolite profiling for identification and stratification of pheochromocytomas/ paragangliomas due to succinate dehydrogenase deficiency. Journal of Clinical Endocrinology and Metabolism 201499 3903-3911. (https://doi. org/10.1210/jc.2014-2151)

16 Imperiale A, Moussallieh FM, Roche P, Battini S, Cicek AE, Sebag F, Brunaud L, Barlier A, Elbayed K, Loundou A et al. Metabolome profiling by HRMAS NMR spectroscopy of pheochromocytomas and paragangliomas detects SDH deficiency: clinical and pathophysiological implications. Neoplasia 201517 55-65. (https:// doi.org/10.1016/j.neo.2014.10.010)

17 Rao JU, Engelke UF, Sweep FC, Pacak K, Kusters B, Goudswaard AG, Hermus AR, Mensenkamp AR, Eisenhofer G, Qin N et al. Genotype-specific differences in the tumor metabolite profile of pheochromocytoma and paraganglioma using untargeted and targeted metabolomics. Journal of Clinical Endocrinology and Metabolism 2015100 E214-E222. (https://doi.org/10.1210/jc.2014-2138)

18 Sun N, Wu Y, Nanba K, Sbiera S, Kircher S, Kunzke T, Aichler M, Berezowska S, Reibetanz J, Rainey WE et al. High resolution tissue mass spectrometry imaging reveals a refined functional anatomy of the human adult adrenal gland. Endocrinology 2018159 1511-1524. (https://doi.org/10.1210/en.2018-00064)

19 Papathomas TG \& Nose V. New and emerging biomarkers in endocrine pathology. Advances in Anatomic Pathology 201926 198-209. (https:// doi.org/10.1097/PAP.0000000000000227)

20 Sun N, Kunzke T, Sbiera S, Kircher S, Feuchtinger A, Aichler M, Herterich S, Ronchi CL, Weigand I, Schlegel N et al. Prognostic relevance of steroid sulfation in adrenocortical carcinoma revealed by molecular phenotyping using high-resolution mass spectrometry imaging. Clinical Chemistry 201965 1276-1286. (https://doi. org/10.1373/clinchem.2019.306043)

21 Murakami M, Rhayem Y, Kunzke T, Sun N, Feuchtinger A, Ludwig P, Strom TM, Gomez-Sanchez C, Knosel T, Kirchner T et al. In situ metabolomics of aldosterone-producing adenomas. JCI Insight 20194 (https://doi.org/10.1172/jci.insight.130356)

22 Guijas C, Montenegro-Burke JR, Domingo-Almenara X, Palermo A, Warth B, Hermann G, Koellensperger G, Huan T, Uritboonthai W, Aisporna AE et al. Metlin: a technology platform for identifying knowns and unknowns. Analytical Chemistry 201890 3156-3164. (https://doi.org/10.1021/acs.analchem.7b04424)

23 Wishart DS, Feunang YD, Marcu A, Guo AC, Liang K, VazquezFresno R, Sajed T, Johnson D, Li C, Karu N et al. HMDB 4.0: the 
human metabolome database for 2018. Nucleic Acids Research 201846 D608-D617. (https://doi.org/10.1093/nar/gkx1089)

24 Palmer A, Phapale P, Chernyavsky I, Lavigne R, Fay D, Tarasov A, Kovalev V, Fuchser J, Nikolenko S, Pineau C et al. FDR-controlled metabolite annotation for high-resolution imaging mass spectrometry. Nature Methods 201714 57-60. (https://doi.org/10.1038/ nmeth.4072)

25 Hopewell R \& Ziff EB. The nerve growth factor-responsive PC12 cell line does not express the Myc dimerization partner Max. Molecular and Cellular Biology 199515 3470-3478. (https://doi.org/10.1128/ mcb.15.7.3470)

26 Comino-Mendez I, Gracia-Aznarez FJ, Schiavi F, Landa I, LeandroGarcia LJ, Leton R, Honrado E, Ramos-Medina R, Caronia D, Pita G et al. Exome sequencing identifies MAX mutations as a cause of hereditary pheochromocytoma. Nature Genetics 201143 663-667. (https://doi.org/10.1038/ng.861)

27 Favier J, Briere JJ, Burnichon N, Riviere J, Vescovo L, Benit P, GiscosDouriez I, De Reynies A, Bertherat J, Badoual C et al. The Warburg effect is genetically determined in inherited pheochromocytomas. PLOS ONE 20094 e7094. (https://doi.org/10.1371/journal. pone.0007094)

28 Rao JU, Engelke UF, Rodenburg RJ, Wevers RA, Pacak K, Eisenhofer G, Qin N, Kusters B, Goudswaard AG, Lenders JW et al. Genotypespecific abnormalities in mitochondrial function associate with distinct profiles of energy metabolism and catecholamine content in pheochromocytoma and paraganglioma. Clinical Cancer Research 201319 3787-3795. (https://doi.org/10.1158/1078-0432.CCR-123922)

29 Imperiale A, Moussallieh FM, Sebag F, Brunaud L, Barlier A, Elbayed K, Bachellier P, Goichot B, Pacak K, Namer IJ et al. A new specific succinate-glutamate metabolomic hallmark in SDHx-related paragangliomas. PLoS ONE 20138 e80539. (https://doi.org/10.1371/ journal.pone.0080539)

30 Eisenhofer G, Klink B, Richter S, Lenders JW \& Robledo M. Metabologenomics of phaeochromocytoma and paraganglioma: an integrated approach for personalised biochemical and genetic testing. Clinical Biochemist Reviews 201738 69-100.

31 Rodrigues E \& Macauley MS. Hypersialylation in cancer: Modulation of inflammation and therapeutic opportunities. Cancers 201810207. (https://doi.org/10.3390/cancers10060207)

32 Job S, Draskovic I, Burnichon N, Buffet A, Cros J, Lepine C, Venisse A, Robidel E, Verkarre V, Meatchi T et al. Telomerase activation and ATRX mutations are independent risk factors for metastatic pheochromocytoma and paraganglioma. Clinical Cancer Research 2019 25 760-770. (https://doi.org/10.1158/1078-0432.CCR-18-0139)

33 Calsina B, Castro-Vega LJ, Torres-Perez R, Inglada-Perez L, CurrasFreixes M, Roldan-Romero JM, Mancikova V, Leton R, Remacha L, Santos $\mathrm{M}$ et al. Integrative multi-omics analysis identifies a prognostic miRNA signature and a targetable miR-21-3p/TSC2/mTOR axis in metastatic pheochromocytoma/paraganglioma. Theranostics 20199 4946-4958. (https://doi.org/10.7150/thno.35458)

34 Ala M. The footprint of kynurenine pathway in every cancer: A new target for chemotherapy. European Journal of Pharmacology 2021896 173921. (https://doi.org/10.1016/j.ejphar.2021.173921)

35 Walczak K, Zurawska M, Kis J, Starownik R, Zgrajka W, Bar K, Turski WA $\&$ Rzeski W. Kynurenic acid in human renal cell carcinoma: Its antiproliferative and antimigrative action on Caki-2 cells. Amino Acids 201243 1663-1670. (https://doi.org/10.1007/s00726-012-1247-5)

36 Walczak K, Turski WA \& Rzeski W. Kynurenic acid enhances expression of p21 Waf1/Cip1 in colon cancer HT-29 cells. Pharmacological Reports 201264 745-750. (https://doi.org/10.1016/ s1734-1140(12)70870-8)

Received 8 December 2020

Revised version received 31 March 2021

Accepted 12 May 2021 